\title{
Estimation of Hybrid Systems Using Discrete Sensors
}

\author{
Xenofon D. Koutsoukos \\ Department of Electrical Engineering and \\ Computer Science \\ Vanderbilt University \\ Nashville, TN 37235, USA \\ Xenofon. Koutsoukos@vanderbilt.edu
}

\begin{abstract}
State estimation of hybrid systems is a significant problem for the design of feedback control and model-based diagnosis algorithms. In this paper, a methodology for state estimation of hybrid systems with discrete sensors based on particle filtering is presented. The quality of the algorithm is evaluated by comparing its performance with Cramér-Rao bounds computed for the discrete-time hybrid filtering problem. The approach is illustrated using simulation results of a tank system example.
\end{abstract}

\section{INTRODUCTION}

The ability of estimating the state of a hybrid system is critical for designing feedback control and model-based diagnosis algorithms for embedded systems. The state estimation problem for hybrid systems presents interesting new challenges. Hybrid estimation algorithms require keeping track of multiple models and the transitions between them that is computationally very expensive. In addition, hybrid systems may contain both discrete and continuous observations that require discrete and continuous observers to be appropriately combined in order to estimate the full hybrid state.

In this paper, we focus on a significant class of hybrid systems that have only discrete sensors. Today's discrete sensors can accurately measure many different things, are easy to instrument, provide built-in communication capabilities, and still are very cheap. Discrete sensors are widely used in hybrid system applications to measure continuous quantities. Photoelectric sensors, for example, are used to detect paper edges in printers, fluid levels in chemical processes, and as proximity sensors in autonomous robotic applications.

Discrete sensors are particularly suitable for monitoring guard conditions that trigger mode transitions in hybrid systems. However, they do not provide any information between consecutive measurements. The objective of this paper is to investigate how the full state can be estimated using only discrete observations. The contribution of the paper is twofold. First, we present an algorithm for state estimation of hybrid systems with discrete sensors based on particle filtering. Second, we evaluate the estimation algorithm by comparing its performance with Cramér-Rao bounds computed for the discrete-time hybrid filtering problem.

Several approaches for estimation of hybrid systems have been recently proposed. A method based on banks of extended Kalman filters has been presented in [5] where only trajectories with high confidence probability are traced. An approach using both discrete and continuous observers based on finite state machines and linear systems has been proposed in [1]. Sequential Monte Carlo (or particle filtering) methods can support process densities that contain both continuous and discrete dynamics and have been explored for hybrid diagnosis in [11]. However, autonomous transitions between modes triggered by the continuous dynamics have not been considered. Particle filtering has been applied also for a class of hybrid systems modeled by dynamic Bayesian networks in [8] where the autonomous transitions between discrete states are defined using the so-called softmax conditional probability distributions. A Bayesian approach for mode estimation of hybrid systems has been presented in [15] and has been demonstrated for monitoring and diagnosis of electro-mechanical systems. This approach uses prior from a temporal discrete event model, discrete measurements from LEDs and continuous measurements from acoustic and current sensors. Finally, the particle filtering based algorithm presented in this paper has been applied in the case of continuous measurements in [10]. A distributed version of the algorithm applied to a cryogenic propulsion system can be found in [9].

Evaluation of estimation algorithms is usually carried out by comparing their performance with Cramér-Rao (CR) bounds. CR bounds for discrete-time nonlinear stochastic dynamic systems have been studied extensively, see for example [7] and the references therein. In this paper, we use the CR bounds derived in [12] and also presented in [14]. In particular, this paper presents an approach for quantifying the performance of the hybrid estimation algorithm based on the Cramér-Rao (CR) bound for discrete-time nonlinear filtering. The CR bound defined as the inverse of the Fisher Information Matrix (FIM) provides a lower bound for the error covariance matrix. A computational technique that computes the CR bound for hybrid dynamical systems with discrete measurements is presented. The performance of the particle filtering approach is evaluated by comparing the relative proximity of the mean square error matrix with the CR bounds using multiple simulation experiments. It should be noted that the approach is based on the computation of CR bounds for discrete-time nonlinear stochastic systems and is not limited to the case of discrete observations.

The paper is organized as follows. The problem of state estimation for hybrid systems with discrete sensors is stated 
in Section II. The state estimation approach based on particle filtering is presented in Section III. A CR bound based method for the performance evaluation of the algorithnm is described in Section IV. The approach is illustrated with simulation results of a tank system example in Section V. Discussion and future work are presented in Section VI.

\section{PROBLEM STATEMENT}

Hybrid systems contain interacting discrete and continuous dynamics. The discrete dynamics are usually described by discrete event models with a finite state space $Q$. Every discrete state (or mode) $q$ corresponds to a unique differential (difference) equation $\dot{x}=f(q, x)\left(x_{t+1}=f\left(q_{t}, x_{t}\right)\right), x \in$ $\Re^{n}$ that governs the continuous dynamics. The state of the hybrid system is given by $s=(q, x)$. The state can change either by time delay as described by the differential/difference equation or by a transition. Mode transitions $e=\left(q_{1}, q_{2}\right)$ may occur either upon receiving an external control command or when the continuous state satisfies a guard $x \in G(e)$ that labels the transition. Mode transitions that depend on the continuous behavior of the system are called autonomous.

In practical applications, the state $s=(q, x)$ has to be reconstructed from the observations. In this paper, we assume that only observations from an array of discrete sensors $S=\left\{S_{1}, S_{2}, \ldots, S_{m}\right\}, m \geq n$ that quantizes the state space are available. Each sensor $S_{i}$ is associated with a single continuous state $x_{i_{j}} \in \Re, i_{j}=1,2, \ldots, n$. Let $z_{i} \in \Re$ be the prespecified level of sensor $S_{i}$. Then the output of $S_{i}$ is defined as

$$
y_{i}=\left\{\begin{array}{ll}
1, & \text { if } x_{i_{j}} \geq z_{i} \\
0, & \text { if } x_{i_{j}}<z_{i}
\end{array} .\right.
$$

Discrete sensors such as photoelectric sensors typically use a digital clock. The continuous state $x_{i_{j}}$ may exceed or fall below $z_{i}$ between two consecutive clock ticks. If the value of the sensor $S_{i}$ changes at time $t=0,1,2, \ldots$, then we assume that $x_{i_{j}}(t) \approx z_{i}$. Therefore, we can model such a discrete sensor as

$$
y_{i}(t)= \begin{cases}z_{i}+\xi_{i}(t), & \text { if } y_{i}(t) \neq y_{i}(t-1) \\ \text { null }, & \text { otherwise }\end{cases}
$$

where $\xi_{i}(t)$ is zero mean Gaussian noise. The noise term models the difference between $z_{i}$ and the value of the state $x_{i_{j}}$ at time instant $t$, which is the first clock tick after $x_{i_{j}}$ has crossed $z_{i}$. It is assumed that at every time instant, at most $n$ sensor values may change from 0 to 1 . Let $y_{t}$ denote the measurements vector, then the observation model is given by

$$
y_{t}=H_{t} x_{t}+\xi_{t}
$$

where $H_{t}$ is a matrix of variable dimension $m_{t} \times n, 0 \leq$ $m_{t} \leq n$. If $m_{t}=0$ then $H_{t}$ is the null matrix. If $m_{t} \neq 0$ then each row of $H_{t}$ consists of 0 's and exactly one 1 at the $j^{\text {th }}$ position corresponding to the state $x_{j}$.

In this paper, the estimation problem is formulated using a Bayesian approach based on a discrete-time representation of the system dynamics. The continuous dynamics of the system are described by the discrete-time model

$$
\begin{aligned}
x_{t+1} & =f_{q_{t}}\left(x_{t}, u_{t}\right)+\nu_{t} \\
y_{t} & =H_{t} x_{t}+\xi_{t}
\end{aligned}
$$

and $\nu_{t}$ and $\xi_{t}$ denote process and measurement noise respectively. The evolution of the discrete state can be described by the transition function

$$
q_{t+1}=\delta\left(q_{t}, \sigma_{t}, x_{t}\right)
$$

where $\sigma_{t}$ denotes events corresponding to the control commands. The hybrid estimation problem is to compute the most likely hybrid state $s_{t}=\left(q_{t}, x_{t}\right)$ given the history of observations $Y_{t}=\left(y_{t_{1}}, y_{t_{2}}, \ldots\right)$ that denotes the discrete measurements up to time $t$, the sequence of continuous control inputs $U_{t}=\left(u_{0}, u_{1}, \ldots, u_{t}\right)$, and the history of control events $\left(\sigma_{t_{1}}, \sigma_{t_{2}}, \ldots\right)$ up to time $t$.

\section{HYBRID SYSTEM ESTIMATION USING PARTICLE FILTERING}

A challenging aspect of hybrid estimation is monitoring the autonomous mode transitions and using the appropriate mode $q$ for updating the estimate of the continuous state $x$. Discrete sensors are very suitable for monitoring guard conditions that trigger such autonomous transitions. However, such sensors result in loss of information for the continuous state. In this paper, an algorithm for estimating both discrete and continuous state based only on the discrete measurements is presented. The general idea of the algorithm is that at every time step the mode transition probability can be evaluated using the current estimate of the continuous state. Given these transition probabilities, the algorithm focuses on the most likely modes and updates the continuous estimate. If there are new observations the continuous state is updated by conditioning on these observations. If there are no observations, the continuous state is updated by diffusing the previous belief state using the system dynamics. Note that the probability of mode transitions triggered by control commands $\sigma_{t}$ can be usually computed by discrete estimation techniques, for example, using hidden Markov models and will not be considered.

Formally, the estimation algorithm is based on the following decomposition of the process density

$$
p\left(q_{t}, x_{t} \mid q_{t-1}, x_{t-1}\right)=p\left(x_{t} \mid q_{t}, q_{t-1}, x_{t-1}\right) P\left(q_{t} \mid q_{t-1}, x_{t-1}\right)
$$

where the density $p\left(x_{t} \mid q_{t}, q_{t-1}, x_{t-1}\right)$ describes the evolution of the continuous state conditioned on the mode and the distribution $P\left(q_{t} \mid q_{t-1}, x_{t-1}\right)$ describes the mode transition probability conditioned on the continuous state. We define the mode transition probability matrix with elements $T_{i j}(t)=$ $p\left(q_{t}=j \mid x_{t-1}, q_{t-1}=i\right), i, j=1, \ldots,|Q|$. Let $G_{i j}$ be the guard corresponding to the transition from mode $i$ to mode $j$. Assuming that the system is at mode $q_{i}$ and that the probability of the transition $q_{i} \rightarrow q_{j}$ is equal to the 
probability the guard $G_{i j}$ is satisfied, the mode transition probability matrix can be computed as

$$
T_{i j}(t)=\int_{G_{i j}} p\left(x_{t-1} \mid Y_{t-1}, U_{t-1}, q_{t-1}=i\right) d x_{t-1}
$$

where $p\left(x_{t-1} \mid Y_{t-1}, U_{t-1}, q_{t-1}=i\right)$ is the conditional density of the continuous state at time $t-1$ and $Y_{t-1}$ denote the observation history up to time $t-1$. It should be noted that the performance of the algorithm can be improved by transforming the guard conditions so that they form a cover of the state space, details can be found in [10].

Next, we describe a particle filtering algorithm that allows the computation of the complex integrals that represent the mode transition probabilities. The transition probabilities are then used to dynamically assign particles to the discrete modes, thus focusing on the most likely transitions. The belief for the continuous state is then updated by applying the drift due to the deterministic component and the diffusion due to the random component of the system dynamics. If there are no observations at the current time step, the algorithm proceeds with prediction of the next state. If there are available measurements from the discrete sensors, the belief is updated by taking into consideration the likelihood function. In the following, it is assumed without loss of generality that there are no control inputs $u_{t}$ in order to simplify the notation. Detailed descriptions of particle filtering methods for estimation of dynamical systems can be found in [3].

Let $\left\{s_{t-1}^{(k)}, w_{t-1}^{(k)}, k=1, \ldots, N\right\}$ denote the sample set at time $t-1$ where $s_{t-1}^{(k)}=\left(q_{t-1}^{(k)}, x_{t-1}^{(k)}\right)$ is the $k^{t h}$ sample of the local hybrid state and $w_{t-1}^{(k)}$ its probability weight. The $k^{\text {th }}$ sample of the predicted state at time $t$ is denoted by $\tilde{s}_{t}^{(k)}=\left(\tilde{q}_{t}^{(k)}, \tilde{x}_{t}^{(k)}\right)$. The estimation algorithm consists of the following steps:

Initialization $t=0$.

sample $s_{0}^{(k)}=\left(q_{0}^{(k)}, x_{0}^{(k)}\right), k=1,2, \ldots, N$ from

$p\left(q_{0}\right)$ and $p\left(x_{0}\right)$ and set $t=1$.

\section{Prediction}

apply $p\left(s_{t} \mid s_{t-1}^{(k)}\right)$ to compute each $\tilde{s}_{t}^{(k)}$.

i. compute $T_{i j}(t)=p\left(q_{t}=j \mid x_{t-1}, q_{t-1}=i\right)$ from $s_{t-1}^{(k)}=\left(q_{t-1}^{(k)}, x_{t-1}^{(k)}\right)$ and $w_{t-1}^{(k)}$.

ii. sample $\tilde{q}_{t}^{(k)}$ from $T_{i j}(t)$.

iii. apply $p\left(x_{t} \mid x_{t-1}^{(k)}, q_{t-1}^{(k)}, \tilde{q}_{t}^{(k)}\right)$ to compute $\tilde{x}_{t}^{(k)}$.

If there are new observations then

evaluate the importance weights $w_{t}^{(k)}=p\left(y_{t} \mid \tilde{s}_{t}^{(k)}\right)$. normalize the weights.

\section{Resampling}

end resample $N$ particles $s_{t}^{(k)}$ from $\tilde{s}_{t}^{(k)}$.

set $t \leftarrow t+1$ and go to step 2 .

The interaction between the discrete and continuous dynamics is addressed at the prediction step of the algorithm in order to compute the distribution of the predicted state $\tilde{s}_{t}^{(k)}$. Consider that at time $t$ the prediction $p\left(q_{t-1}, x_{t-1} \mid Y_{t-1}\right)$ is represented by the sample set $\left\{q_{t-1}^{(k)}, x_{t-1}^{(k)}, w_{t-1}^{(k)}, k=\right.$ $1, \ldots, N\}$. The mode transition probability matrix can be computed by

$$
T_{i j}(t)= \begin{cases}\frac{\sum_{k \in \hat{G}_{i j}} w_{t-1}^{(k)}}{\sum_{k \in \hat{I}} w_{t-1}^{(k)}}, & i \neq j \\ 1-\sum_{\ell \neq i} T_{i \ell}(t), & i=j\end{cases}
$$

where $k \in \hat{G}_{i j} \Leftrightarrow q_{t-1}^{(k)}=i \wedge x_{t-1}^{(k)} \in G_{i j}$ and $k \in \hat{I} \Leftrightarrow$ $q_{t-1}^{(k)}=i$.

The mode of the system is computed using the particles as the most likely mode at every time step and the continuous state is computed using only particles (after renormalization) from the most likely mode, that is

$$
\hat{q}_{t}=\arg \max _{i} \sum_{k \in \hat{Q}_{i}} w_{t}^{(k)} \text { and } \hat{x}_{t}=\frac{\sum_{k \in \hat{Q}} w_{t}^{(k)} x_{t}^{(k)}}{\sum_{k \in \hat{Q}} w_{t}^{(k)}}
$$

where $\hat{Q}_{i}=\left\{k \mid q_{t}^{(k)}=i\right\}$ and $\hat{Q}=\left\{k \mid q_{t}^{(k)}=\hat{q}_{t}\right\}$. Finally, the mean square error matrix (MSEM) is computed as

$$
\hat{P}_{t}=\frac{\sum_{k \in \hat{Q}} w_{t}^{(k)}\left(\hat{x}_{t}-x_{t}^{(k)}\right)\left(\hat{x}_{t}-x_{t}^{(k)}\right)^{T}}{\sum_{k \in \hat{Q}} w_{t}^{(k)}} .
$$

\section{PERFORMANCE EVALUATION BASED ON CRAMER-RAO BOUNDS}

This section presents an approach for quantifying the performance of the hybrid estimation algorithm based on the Cramér-Rao (CR) bounds for discrete-time nonlinear filtering. Consider the discrete-time dynamical system

$$
\begin{aligned}
x_{t+1} & =f_{q_{t}}\left(x_{t}, u_{t}\right)+\nu_{t} \\
y_{t} & =h_{t}\left(x_{t}\right)+\xi_{t}
\end{aligned}
$$

where $u_{t}$ is a deterministic control input and $\nu_{t}$ and $\xi_{t}$ are zero mean Gaussian process and measurement noises with covariances $Q_{t}$ and $R_{t}$ respectively. The initial state is described as $p\left(x_{0}\right)=\mathcal{N}\left(x_{0}: \mu_{0}, P_{0}\right)$. The evolution of the discrete mode is described by (3). It is assumed that, given $x_{t}$, the mode $q(t)$ is uniquely defined based on the guard conditions of the hybrid system. Then the system (7)(8) can be viewed as a time-varying nonlinear stochastic dynamic system. The transition and measurement densities can be expressed as

$$
\begin{aligned}
p\left(x_{t+1} \mid x_{t}\right) & =\mathcal{N}\left(x_{t+1}: f_{q_{t}}\left(x_{t}, u_{t}\right), Q_{t}\right) \\
p\left(y_{t} \mid x_{t}\right) & =\mathcal{N}\left(y_{t}: h_{t}\left(x_{t}\right), R_{t}\right) .
\end{aligned}
$$

The CR bound defined as the inverse of the Fisher Information Matrix (FIM) provides a lower bound for the error covariance matrix

$$
E\left[\left(\hat{x}_{t}-x\right)\left(\hat{x}_{t}-x\right)^{T}\right] \geq F_{t}^{-1}
$$


where $E[\cdot]$ denotes expectation and $A \geq B$ means that $A-$ $B$ is positive semidefinite. The CR bound can be computed recursively [12], [14] by

$$
F_{t}=Q_{t}^{-1}+L_{t}-\Psi_{t}\left(F_{t-1}+\Phi_{t}\right)^{-1} \Psi_{t}^{T}
$$

where

$$
\begin{aligned}
L_{t} & =E\left[\nabla_{x_{t}} h_{t}^{T}\left(x_{t}\right) R_{t}^{-1} \nabla_{x_{t}} h_{t}\left(x_{t}\right)\right] \\
\Psi_{t} & =E\left[-\nabla_{x_{t}} f_{q_{t}}^{T}\left(x_{t}, u_{t}\right)\right] Q_{t}^{-1} \\
\Phi_{t} & =E\left[\nabla_{x_{t}} f_{q_{t}}^{T}\left(x_{t}, u_{t}\right) Q_{t}^{-1} \nabla_{x_{t}} f_{q_{t}}\left(x_{t}, u_{t}\right)\right] .
\end{aligned}
$$

The recursion is initiated by $F_{0}^{-1}=P_{0}$.

In the case of hybrid systems with discrete observations, the conditional density $p\left(y_{t} \mid x_{t}\right)$ does not exist for every $t$. At time instants when observations are available, the Fisher information matrix and the CR bound can be computed by equation (10). Between observations the Fisher information matrix can be defined as $F_{t}=P_{t}^{-1}$ where $P_{t}$ is the covariance matrix. $P_{t}$ can be computed recursively [6] by

$$
P_{t+1}=E\left[\nabla_{x_{t}} f_{q_{t}}\left(x_{t}, u_{t}\right) P_{t} \nabla_{x_{t}} f_{q_{t}}^{T}\left(x_{t}, u_{t}\right)\right]+Q_{t} .
$$

Practically, the computation of the CR bounds requires the evaluation of the expectations in equations (10) and (11). The CR bounds can be computed by carrying out $M$ simulation experiments to generate $M$ realizations of the state trajectory for some fixed length $T,\left\{x_{t}(j)\right\}_{t=1, \ldots, T}^{j=1, \ldots, M}$, starting at i.i.d. initial positions drawn from $p\left(x_{0}\right)$. Given $x_{0}, q_{0}$ is uniquely defined and the transition density can be described by $\mathcal{N}\left(x_{1}\right.$ : $\left.x_{0}, f_{q_{0}}\left(x_{0}, u_{0}\right)\right)$. The matrices that involve expectations can be replaced by their estimates, for example

$$
\hat{\Psi}_{t}=\frac{1}{M} \sum_{j=1}^{M}\left[-\left.\nabla_{x_{t}} f_{q_{t}}\left(x_{t}, u_{t}\right)\right|_{x_{t}=x_{t}(j)}\right] Q_{t}^{-1} .
$$

Clearly, $M$ simulation experiments will not generate discrete observations at the same time instants. Let $M_{t}^{o b s}\left(M_{t}^{\overline{o b s}}\right)$ denote the number of experiments for which there exists (there does not exist) a discrete observation at time $t$. The FIM at time $t$ and for the $j^{t h}$ simulation experiment is denoted by $\hat{F}_{t}^{o b s}(j)$ if it is computed by equation (10) and $\hat{F}_{t}^{\overline{o b s}}(j)$ if it is computed by equation (11). The estimated of the FIM is then computed (after renumbering of the simulation experiments) as

$$
\hat{F}_{t}=\frac{M_{t}^{o b s}}{M} \sum_{j=1}^{M_{t}^{o b s}} \hat{F}_{t}^{o b s}(j)+\frac{M_{t}^{\overline{o b s}}}{M} \sum_{j=1}^{\overline{M_{t}^{o b s}}} \hat{F}_{t}^{\overline{o b s}}(j) .
$$

The quality of the estimation algorithm can be evaluated based on the inequality (9). The scalar inequality corresponding to the mean square error given by

$$
\frac{1}{M} \sum_{i=1}^{M}\left\|\hat{x}_{t}^{(i)}-x_{t}^{(i)}\right\|_{2}^{2} \geq \operatorname{tr}\left(\hat{F}_{t}^{-1}\right)
$$

is a convenient way for quantifying the filter performance. Evaluating this inequality requires the application of the estimation algorithm and the computation of the CR bounds for $M$ i.i.d. state trajectories.

\section{A TANK SYSTEM EXAMPLE}

A tank system example is used to demonstrate the state estimation approach. This example has been proposed as a benchmark for control reconfiguration in [4] and has been used for illustrating estimation, fault detection, and control reconfiguration methods for hybrid systems in [2], [13]. The system consists of two identical cylindrical tanks that are connected by a pipe at level $h$, as shown in figure 1 . We denote by $h_{1}$ and $h_{2}$ the water levels in tanks 1 and 2 respectively. The input flow $Q_{i n}$ is provided by a pump and it is described by $Q_{i n}=V_{i n} k_{i n} u(t)$, where $V_{i n} \in\{0,1\}$ represents a valve that can be used to turn on or off the pump, $k_{i n}$ is a linear gain, and $u(t)$ is the input signal. The flow $Q_{a}$ between the two tanks is controlled by a valve $V_{a}$. An outlet valve $V_{\text {out }}$ located at the bottom of tank 2 is used to empty the tank. Tank 2 is equipped with a sensor that measures the output flow which is described by $Q_{\text {out }}=V_{\text {out }} k_{\text {out }} \sqrt{\rho g h_{2}}$ where $V_{\text {out }} \in\{0,1\}$ represents the outlet valve, $k_{\text {out }}$ is a linear gain, $\rho$ is the density of the water, and $g$ is the gravity constant. The system's dynamic evolution is described by

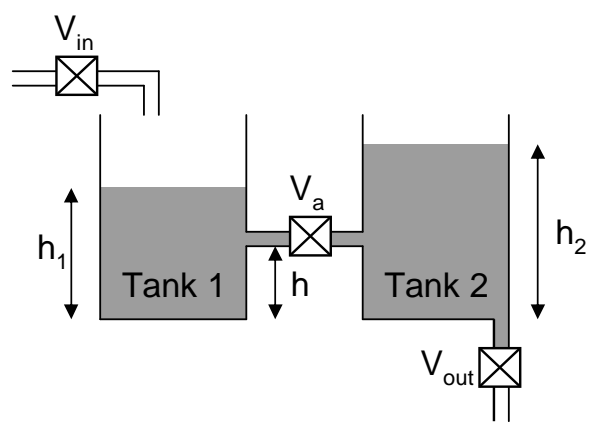

Fig. 1. Two-tank system

$$
\begin{aligned}
& \dot{h}_{1}=\frac{1}{A}\left(Q_{\text {in }}-Q_{a}\right) \\
& \dot{h}_{2}=\frac{1}{A}\left(Q_{a}-Q_{\text {out }}\right)
\end{aligned}
$$

where $A$ is the section of each cylindrical tank. There are four modes of behavior for the flow $Q_{a}$ which depend on the water levels $h_{1}$ and $h_{2}$ as follows:

$Q_{a}= \begin{cases}0, & h_{1}<h, h_{2}<h \\ V_{a} k_{a} \sqrt{\rho g\left(h_{1}-h\right)}, & h_{1}>h, h_{2}<h \\ -V_{a} k_{a} \sqrt{\rho g\left(h_{2}-h\right)}, & h_{1}<h, h_{2}>h \\ \operatorname{sign}\left(h_{1}-h_{2}\right) V_{a} k_{a} \sqrt{\rho g\left|h_{1}-h_{2}\right|}, & h_{1}>h, h_{2}>h\end{cases}$

where $V_{a} \in\{0,1\}$ and $k_{a}$ is a linear gain. The evolution of the continuous state $x=\left[h_{1}, h_{2}\right]^{T}$ can be described by

$$
\dot{x}=f_{q}(x(t), u(t))+\nu(t)
$$


where $q \in\{1,2,3,4\}$ is the discrete mode and $\nu(t)$ is process noise. For every mode, we have a set of ordinary differential equations and the system transitions between modes based on $x$ as described by (13). Clearly, these transitions are autonomous since they depend on the continuous behavior of the system as described by the guard conditions.

We assume the following values for the parameters of the system. All valves are open $V_{\text {in }}=V_{a}=V_{\text {out }}=1, h=.3 \mathrm{~m}$, $k_{\text {in }}=.08, k_{a}=.001, k_{\text {out }}=.001, g=9.81 \mathrm{~m} / \mathrm{sec}^{2}$, $\rho=1000$, and $A=0.0154 \mathrm{~m}^{2}$. An array of eight discrete sensors is used to monitor the water level in the tanks. The sensors are placed at $z_{i} \in\{.1, .3, .5, .7\}$ at each tank. Note that there are two sensors at $h=.3$ that monitor the guard conditions of the system. Placing sensors that monitor the guard conditions is not required by the estimation but it improves the performance considerably since more particles are drawn for the current mode at every step of the algorithm. Finally, the process and noise covariance are assumed to be $R_{t}=Q_{t}=\operatorname{diag}(.001)$.

The observation history is generated using a MATLAB/SIMULINK model of the system. The algorithm estimates the the mode of the system and the water levels given the input flow and the discrete observations shown in figure 2. It is assumed that the only information available for the water levels is provided by the discrete sensors. Initially, $x_{0}$ is drawn from a uniform distribution assuming that $.1 \leq h_{1}<$ .3 and $.7 \leq h_{2}<1$. The mode is $q_{0}=3$ for every sample $x_{0}$. The estimated hybrid state of the system, computed using (5), is shown in figure 3 for $N=200$ particles. As it can be seen by comparing the simulated and estimated trajectories the algorithm is able to track the hybrid state of the system. The mean square error is shown in 4 . The performance of the estimation algorithm for the tank system is evaluated using the inequality (12) for $M=100$ simulation experiments. The particle filter algorithms is initialized for each experiments by drawing $N=200$ particles from a uniform distribution as described above. The algorithm for the computation of the CR bound for each experiment is initialized by the MSEM of the initial samples. Figure 5 illustrates the performance of the particle filtering algorithm.

\section{DISCUSSION AND FUTURE WORK}

Particle filtering algorithms are suitable for estimation of hybrid systems. Such algorithms are very general and straightforward to implement. Computationally, their performance depends on the sample size. Estimation of hybrid systems requires keeping track of multiple models and is based on a relatively large sample size. Determining and controlling an efficient sample size is a significant problem. Additional research is also needed for demonstrating the applicability of particle filtering based estimation algorithms for feedback control and model-based diagnosis of hybrid systems. In the case of discrete observations, specifically, continuous control between observations would be always
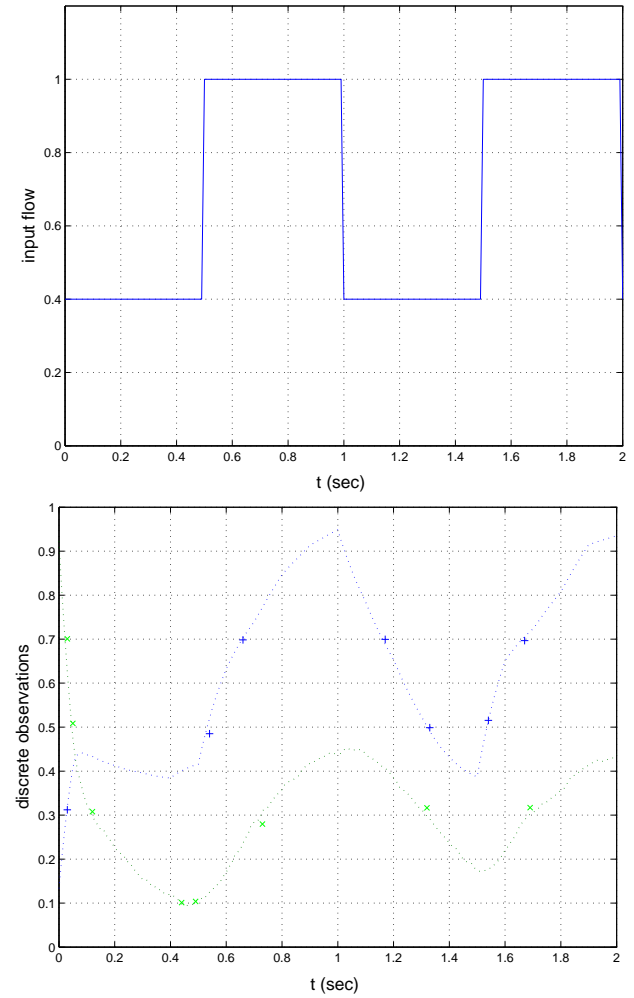

Fig. 2. Input fbw and discrete observations for the tank system

open loop. Particle filters offer a simple way to encapsulate the information provided by the observation history. In addition, the computational performance obtained by recent advances in sequential Monte Carlo techniques allow realtime implementations of such algorithms for a large class of practical applications. Fault diagnosis requires estimating both the state of the system and fault parameters. Smoothing algorithms that consider present and future measurements seem promising. CR bounds can be also derived and can be used to characterize diagnosability properties. Finally, the techniques used for the computation of the CR bounds can be useful for placement of discrete sensors. Sensor locations can be determimed using Monte Carlo simulations by minimizing the inverse Fisher information matrix.

\section{ACKNOWLEDGMENTS}

The author would like acknowledge the partial support by Xerox Foundation for this work and to thank Feng Zhao and James Kurien for useful discussions and suggestions concering the particle filtering algorithms.

\section{REFERENCES}

[1] A. Balluchi, L. Benvenuti, M. D. Benedetto, and A. Sangiovanni-Vincentelli. Design of observers for hybrid systems. In Hybrid Systems: Computation and Control (HSCC'02), Vol. 2289, LNCS, pages 76-89. Springer, 2002. 

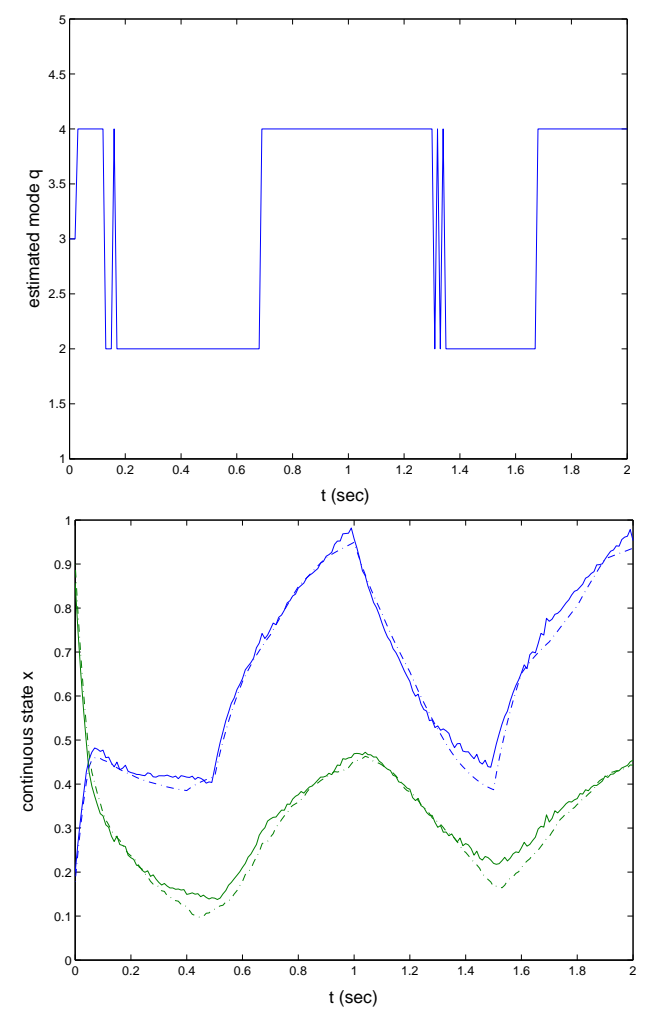

Fig. 3. Estimated mode and continuous state for the tank system (the dashed line shows the simulated trajectory)

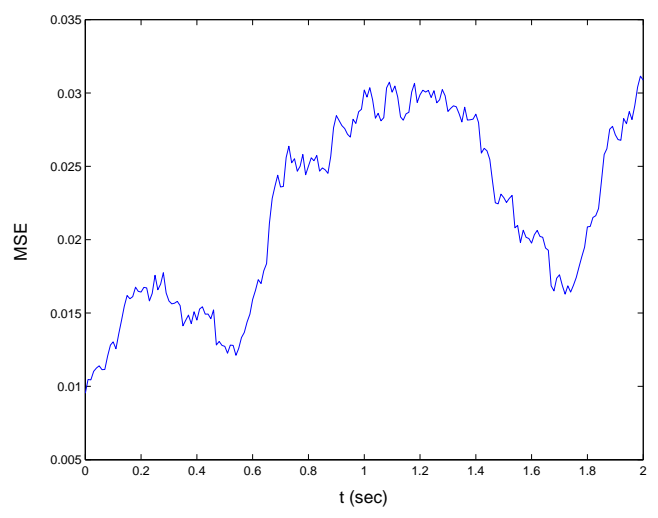

Fig. 4. Mean square error for the tank system

[2] A. Bemporad, D. Mignone, and M. Morari. Moving horizon estimation for hybrid systems and fault detection. In Proc. of the American Control Conference, pages 2471-2475, San Diego, CA, 1999.

[3] A. Doucet, N. D. Freitas, and N. Gordon, editors. Sequential Monte Carlo Methods in Practice. Statistics for Engineering and Information Science. Springer, 2001.

[4] B. Heiming and J. Lunze. Definition of the three tank benchmark problem for controller reconfiguration. In European Control Conference, 1999.

[5] M. Hofbaur and B. Williams. Mode estimation of

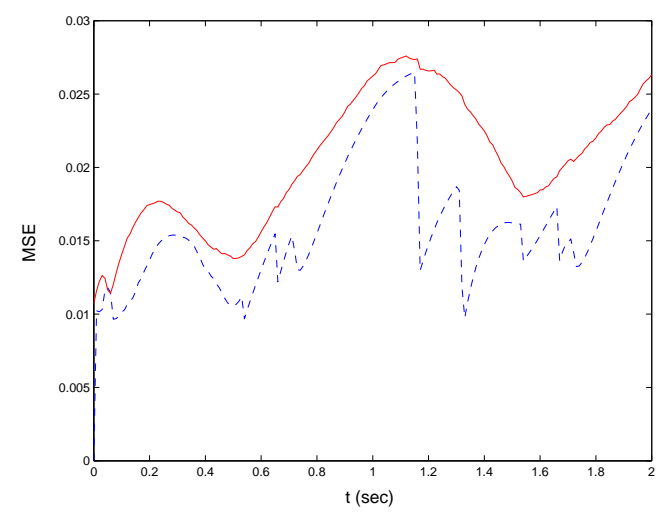

Fig. 5. Mean square error (solid line) and Cramér-Rao bound (dashed line) for the tank system

probabilistic hybrid systems. In Hybrid Systems: Computation and Control (HSCC'02), Vol. 2289, LNCS, pages 253-266. Springer, 2002.

[6] A. Jazwinski. Stochastic Processes and Filtering Theory. Academic Press, 1970.

[7] T. Kerr. Status of CR-like lower bounds for nonlinear filtering. IEEE Trans. on Aerospace and Electronic Systems, 25(5):590-601, 1989.

[8] D. Koller and U. Lerner. Sampling in factored dynamic systems. In Doucet et al. [3], pages 445-464.

[9] X. Koutsoukos, J. Kurien, and F. Zhao. Estimation of distributed hybrid systems using particle filtering methods. In Hybrid Systems: Computation and Control (HSCC'03), Vol. 2623, LNCS, pages 298-313, Springer, 2003

[10] X. Koutsoukos, J. Kurien, and F. Zhao. Estimation of hybrid systems using particle filtering methods. In Proc. of MTNS 2002, Notre Dame, IN, August 2002.

[11] S. McIlraith, G. Biswas, D. Clancy, and V. Gupta. Hybrid systems diagnosis. In Hybrid Systems: Computation and Control, Vol. 1790, LNCS, pages 282-295. Springer, 2000.

[12] P. Tichavský, C. Muravchic, and A. Nehorai. Posterior Cramér-Rao bounds for discrete-time filtering. IEEE Trans. on Signal Processing, 46(5):1386-1396, 1998.

[13] K. Tsuda, D. M. G. Ferrari-Trecate, and M. Morari. Reconfiguration strategies for hybrid systems. In Proc. of the American Control Conference, pages 868-873, Arlington, VA, 2001.

[14] M. Šimandl, J. Královec, and P. Tichavský. Filtering, predictive, and smoothing Cramér-Rao bounds for discrete-time nonlinear dynamic systems. Automatica, 37:1703-1716, 2001.

[15] F. Zhao, X. Koutsoukos, H. Haussecker, J. Reich, P. Cheung, and C. Picardi. Distributed monitoring of hybrid systems: A model-directed approach. In Proc. IJCAI'2001, pages 557-564, Seattle, WA, 2001. 